\title{
Frequency of exacerbations in patients with chronic obstructive pulmonary disease: an analysis of the SPIROMICS cohort
}

\author{
MeiLan K Han, Pedro M Quibrera, Elizabeth E Carretta, R Graham Barr, Eugene R Bleecker, Russell P Bowler, Christopher B Cooper, \\ Alejandro Comellas, David J Couper, Jeffrey L Curtis, Gerard Criner, Mark T Dransfield, Nadia N Hansel, Eric A Hoffman, Richard E Kanner, \\ Jerry A Krishnan, Carlos H Martinez, Cheryl B Pirozzi, Wanda K O'Neal, Stephen Rennard, Donald P Tashkin, Jadwiga A Wedzicha, \\ Prescott Woodruff, Robert Paine III*, Fernando J Martinez*, for the SPIROMICS investigators $\dagger$
}

\section{Summary}

Background Present treatment strategies to stratify exacerbation risk in patients with chronic obstructive pulmonary disease (COPD) rely on a history of two or more events in the previous year. We aimed to understand year to year variability in exacerbations and factors associated with consistent exacerbations over time.

Methods In this longitudinal, prospective analysis of exacerbations in the Subpopulations and Intermediate Outcome Measures in COPD Study (SPIROMICS) cohort, we analysed patients aged 40-80 years with COPD for whom 3 years of prospective data were available, identified through various means including care at academic and non-academic medical centres, word of mouth, and existing patient registries. Participants were enrolled in the study between Nov 12, 2010, and July 31, 2015. We classified patients according to yearly exacerbation frequency: no exacerbations in any year; one exacerbation in every year during 3 years of follow-up; and those with inconsistent exacerbations (individuals who had both years with exacerbations and years without during the 3 years of follow-up). Participants were characterised by the Global Initiative for Chronic Obstructive Lung Disease (GOLD) spirometric category (1-4) on the basis of post-bronchodilator $\mathrm{FEV}_{1}$. Stepwise logistic regression was used to compare factors associated with one or more acute exacerbations of COPD every year for 3 years versus no exacerbations in the same timeframe. Additionally, a stepwise zero-inflated negative binomial model was used to assess predictors of exacerbation count during follow-up in all patients with available data. Baseline symptom burden was assessed with the COPD assessment test. This trial is registered with ClinicalTrials.gov, number NCT01969344.

Findings 2981 patients were enrolled during the study. 1843 patients had COPD, of which 1105 patients had 3 years of complete, prospective follow-up data. $538(49 \%)$ of 1105 patients had at least one acute exacerbation during the 3 years of follow-up, whereas $567(51 \%)$ had none. 82 (7\%) of 1105 patients had at least one acute exacerbation each year, whereas only $23(2 \%)$ had two or more acute exacerbations in each year. An inconsistent pattern (both years with and without acute exacerbations) was common (456 [41\%] of the group), particularly among GOLD stages 3 and 4 patients (256 [56\%] of 456). In logistic regression, consistent acute exacerbations ( $\geq 1$ event per year for 3 years) were associated with higher baseline symptom burden, previous exacerbations, greater evidence of small airway abnormality on CT, lower interleukin-15 concentrations, and higher interleukin- 8 concentrations, than were no acute exacerbations.

Interpretation Although acute exacerbations are common, the exacerbation status of most individuals varies markedly from year to year. Among patients who had any acute exacerbation over 3 years, very few repeatedly had two or more events per year. In addition to symptoms and history of exacerbations in the year before study enrolment, we identified several novel biomarkers associated with consistent exacerbations, including CT-defined small airway abnormality, and interleukin-15 and interleukin-8 concentrations.

Funding National Institutes of Health, and National Heart, Lung, and Blood Institute.

\section{Introduction}

Acute exacerbations of chronic obstructive pulmonary disease (COPD) are important events in the disease course and their effect should not be underestimated. People who have frequent exacerbations have a poor quality of life $\mathrm{e}^{1,2}$ and more rapid decline in lung function compared with those who do not have acute exacerbations. ${ }^{3,4}$ Mortality in the year after an exacerbation requiring hospital admission is estimated to be as high as $21 \% .^{5}$ Treatment for patients with COPD is expensive, with US estimates at nearly $\$ 50$ billion every year in $2007 ;{ }^{6}$ much of this cost is related to acute exacerbation management. Although therapy can reduce exacerbation frequency, ${ }^{7}$ better treatments are still needed. Accordingly, the ability to identify individuals at high risk for the purposes of targeted treatment and research is of paramount importance.

The ECLIPSE investigators suggested that individuals with two or more exacerbations in a given year represent a distinct frequent exacerbator phenotype. ${ }^{8}$ The Global
Lancet Respir Med 2017 5: 619-26 Published Online June 28, 2017 http://dx.doi.org/10.1016/ S2213-2600(17)30207-2 See Comment page 600 ${ }^{*}$ Co-senior authors

†nvestigators listed in the appendix

Division of Pulmonary and Critical Care, Michigan Medicine, Ann Arbor, MI, USA (M K Han MD, Prof J L Curtis MD C H Martinez MD); Department of Biostatistics, University of North Carolina at Chapel Hill, Chapel Hill, NC, USA (PM Quibrera MS, E E Carretta MPH W K O'Neal PhD,

Prof D J Couper PhD); Department of Medicine, Columbia University, New York, NY, USA (Prof R G Barr MD); Center for Genomics and Personalized Medicine Research, Department of Medicine, Wake Forest University, Winston-Salem, NC, USA (Prof E R Bleecker MD); Division of Pulmonary and Critical Care, National Jewish, Denver, $\mathrm{CO}$ USA (Prof R P Bowler MD); Department of Medicine, David Geffen School of Medicine, University of California, Los Angeles, CA, USA (Prof C B Cooper MD, Prof D P Tashkin MD); Division of Pulmonary and Critical Care, University of lowa, lowa City, IA, USA (A Comellas MD); Section of Pulmonary and Critical Care Medicine, Medical Service, VA Ann Arbor Healthcare System, Ann Arbor, MI, USA (Prof L LCurtis); Department of Thoracic Medicine, Temple University, Philadelphia, PA, USA (Prof G Criner MD); Division of Pulmonary and Critical Care, University of Alabama, 
Birmingham, AL, USA (MT Dransfield MD); Department of Medicine, Division of Pulmonary and Critical Care Medicine, Johns Hopkins School of Medicine, Baltimore, MD, USA (N N Hansel MD); Department of Radiology, University of lowa Carver College of Medicine, lowa City, IA, USA (Prof EA Hoffman PhD); Department of Medicine, Division of Respiratory, Critical Care and Occupational

Pulmonary Medicine, University of Utah School of Medicine, Salt Lake City, UT, USA (Prof RE Kanner MD, CB Pirozzi MD,

Prof R Paine III MD); Division of Pulmonary and Critical Care, University of Illinois, Chicago, IL, USA (ProfJ A Krishnan MD); Department of Medicine, University of Nebraska Medical

Center, Omaha, NE, USA (Prof S Rennard MD); Early Clinical Development, AstraZeneca, Cambridge, UK (Prof S Rennard); Department of Respiratory Medicine, Imperial College, London, UK (ProfJ A Wedzicha MD); Cardiovascular Research Institute, Department of Medicine, Division of Pulmonary and Critical Care Medicine, University of California, San Francisco, CA, USA (Prof P Woodruff MD); Section of Pulmonary and Critical Care Medicine, Salt Lake

City Department of Veterans Affairs Medical Center, Salt Lake City, UT, USA (Prof R Paine III); and Division of Pulmonary and Critical Care Medicine,

Department of Medicine, Weill Cornell Medical College, New York, NY, USA (Prof FJ Martinez MD) Correspondence to:

Dr MeiLan K Han, Michigan Medicine, Ann Arbor MI 48109, USA

mrking@med.umich.edu

See Online for appendix

For the SPIROMICS protocol see http://www2.cscc.unc.edu/ spiromics/manuals
Research in context

\section{Evidence before this study}

We searched PubMed between Sept 1, 2010, and Jan 1, 2016 , to identify studies that evaluated frequent exacerbators. We used the search term "frequent" in combination with "COPD" and "exacerbation." The search was limited to human studies published in English. Studies reporting on nine, general population chronic obstructive pulmonary disease (COPD) cohorts were identified that described frequent exacerbator populations. These studies reported a range in prevalence of frequent exacerbators (defined as individuals with two or more exacerbations in any 1 year) between $14 \%$ and $34 \%$. Only one other study, in addition to ECLIPSE, was identified that assessed the stability of the frequent exacerbator phenotype over time, noting exacerbation frequency prior to the study was not a predictor for being a frequent exacerbator during the first year of the study.

\section{Added value of this study}

In this study, we extend the results of ECLIPSE to a patient population with COPD at Global Initiative for Chronic Obstructive Lung Disease (GOLD) stage 1-4. While

Initiative for Chronic Obstructive Lung Disease (GOLD) guide $^{9}$ to COPD diagnosis and management uses a threshold of two or more acute exacerbations in the previous year, or at least one hospital admission related to an acute exacerbation, to identify individuals at high risk of future events (groups C and D). Strategies to prevent exacerbations involve targeting of individuals who are at high risk of future exacerbations, based on the assumption that it is possible to identify a substantial number of these patients prospectively. We aimed to assess the value of the frequent exacerbator classification as first described in ECLIPSE and to understand the factors that are associated with consistent exacerbations over time.

\section{Methods}

\section{Patients and study design}

We did a longitudinal, prospective analysis of acute exacerbations of COPD in the Subpopulations and Intermediate Outcome Measures in COPD Study (SPIROMICS) cohort. SPIROMICS is a multicentre study funded by the National Heart, Lung, and Blood Institute $(\mathrm{NHLBI})^{10}$ and designed to identify COPD subpopulations and to validate intermediate outcome measures. Participants were enrolled between Nov 12, 2010, and July 31, 2015. Aged 40-80 years at enrolment, participants were either healthy never smokers $(\leq 1$ pack-year tobacco smoking history, prebronchodilator $\mathrm{FEV}_{1}$ :forced vital capacity $[\mathrm{FVC}]$ ratio $\geq 0 \cdot 70$, prebronchodilator $\mathrm{FVC}$ at least lower limit of normal, ${ }^{11}$ and without known lung disease or unstable cardiovascular disease) or were current and former smokers of more than 20 pack-years with or without airflow obstruction (defined as postbronchodilator $F E V_{1}$ :FVC ratio of $\geq 0 \cdot 70$ ). Participants were identified approximately half of all patients (538 [49\%] of 1105) had at least one acute exacerbation of COPD during 3 years of follow-up, two or more acute exacerbations in each year were relatively uncommon (23 [2\%] of 1105). Significant variation from year to year in meeting the frequent exacerbator criteria of two or more events per year was noted.

\section{Implications of all the available evidence}

Two or more acute exacerbations in the previous year are part of the criteria for GOLD ABCD grading criteria and have previously been proposed as a key criterion to identify patients for therapeutic trials. However, the data in this Article suggest that a patient's acute exacerbation frequency is subject to substantial fluctuation. Although exacerbation frequency is an important parameter, we show great instability in this measure that potentially limits the clinical value of a threshold of more than two acute exacerbations in the previous year. As such, this criterion might not be the best way to identify individual patients at increased risk of acute exacerbations and subsequently classify individuals for pharmacotherapeutic decision making.

through various means, including care at academic and non-academic medical centres, word of mouth, and existing patient registries (appendix). ${ }^{10}$ The SPIROMICS protocol was approved by the institutional review boards of all participating institutions; all participants gave written informed consent.

Participants were characterised by GOLD spirometric category $1-4,{ }^{12}$ on the basis of spirometric values obtained after each of four inhalations of albuterol $(90 \mu \mathrm{g}$ per inhalation) and ipratropium (18 $\mathrm{\mu g}$ per inhalation). Spirometric tracings were independently reviewed. At the initial study visit, extensive data were collected, including demographics, symptom severity and quality of life, cigarette smoke exposure, spirometry, and 6 min walk distance. High-resolution CT was done according to the study protocol..$^{13}$ Details of this baseline assessment have been previously published. ${ }^{10}$

Self-reported exacerbation data in the year before enrolment were collected at the baseline visit. Prospective exacerbation data were collected every 3 months through a structured telephone questionnaire and three annual clinic visits. Acute exacerbations were defined as events that required health care (ie, office visit, hospital admission, or emergency department visit for a respiratory flare-up) involving the use of antibiotics or systemic corticosteroids, or both. Severe acute exacerbations were defined as those requiring a hospital admission or emergency department visit. Acute exacerbations were managed by the participants' usual care providers; the study did not provide guidance on management.

We measured emphysema and airway wall thickness on high-resolution CT imaging by VIDA software (Apollo version 2.0.003) using a less than -950 Hounsfield unit 
threshold for emphysema and pi10 (square root of wall area of a hypothetical airway with an internal perimeter of $10 \mathrm{~mm}$ ) for airway wall thickening. ${ }^{14}$ Parametric response mapping analysis was done with the Imbio Lung Density Analysis software application (version 2.5) to distinguish regions of emphysema from regions of non-emphysematous gas trapping, indicative of functional small airways disease. ${ }^{15}$

\section{Statistical analysis}

Data analysis was done with SAS software (version 9.4). We compared participants with 3 years of complete acute exacerbation data with the remainder of SPIROMICS participants with less than 3 years of follow-up using twosample $t$ tests for continuous variables and $\chi^{2}$ tests for categorical variables. Two regression models were built. First, among patients with 3 years of follow-up, stepwise logistic regression was used to investigate factors associated with having at least one acute exacerbation in each of the 3 years (consistent acute exacerbations) versus no acute exacerbation in the whole 3 years. Second, a stepwise zero-inflated negative binomial model was used to assess predictors of exacerbation count during follow-up using all patients with available data. Age, sex, race, smoking status, clinical centre of recruitment, and $\mathrm{FEV}_{1}$ percentage predicted were included in all models as potential confounders; follow-up time was included as an offset in this model. For additional variables, a significance level of 0.05 was used as the criterion for entry or deletion at each stage. We considered the following additional predictors: the COPD assessment test (CAT) score, ${ }^{16}$ five measurements obtained from the CT scans (appendix); self-reported history of gastro-oesophageal reflux disease; history of cardiovascular disease; depression and anxiety score from the Hospital Anxiety and Depression Scale questionnaire; previous exacerbation history; blood eosinophil count; white blood cell count; and 12 biologically plausible, circulating biomarkers (appendix). We defined annualised exacerbation rates as the total number of events per person divided by the number of follow-up days for that person, multiplied by 365 . We assessed for collinearity of candidate variables. Many of the imaging variables were correlated with themselves and with $\mathrm{FEV}_{1}$ percentage predicted. Collinearity can be a concern if it makes model estimation unstable, but we did not find that to be the case. Stepwise regression was used to select variables with independently contributing associations after accounting for relevant confounders.

This trial is registered with ClinicalTrials.gov, number NCT01969344.

\section{Role of the funding source}

The funders of the study had no role in study design, data collection, data analysis, data interpretation, or writing of the report. The corresponding author had full access to all the data in the study and had final responsibility for the decision to submit for publication.

\section{Results}

Between Nov 12, 2010, and July 31, 2015, we enrolled 2981 participants; of these, 1843 had COPD and 1105 of these patients had 3 years of complete acute exacerbation data (figure 1). Baseline characteristics of participants, including the extent of airflow obstruction, are shown in table 1. The largest group of patients was at GOLD 2 stage $(\mathrm{n}=494)$, with relatively equal numbers of patients at GOLD $1(n=274)$ and GOLD 3 stages $(n=250)$, followed by GOLD 4 ( $n=87$; appendix). Due to staggered recruitment and protocol-determined termination of data collection, 738 of patients with COPD did not have 3 complete years of exacerbation data (figure 1; appendix). Those with complete 3-year exacerbation data were slightly older, less likely to be current smokers, had a higher $\mathrm{FEV}_{1}$, and had a lower CAT score than patients for whom complete 3-year exacerbation data were not available (appendix).

Among the 1105 patients with complete data, $538(49 \%)$ had at least one acute exacerbation during the 3 years of follow-up, while 567 (51\%) had no exacerbations (table 1; appendix). Exacerbation frequency increased with worsening airflow obstruction (GOLD category; figure 2; appendix). In GOLD categories 3 or 4, most individuals had at least one exacerbation during 3 years of follow-up (165 [66\%] and 73 [84\%], respectively). Overall, 268 (50\%) of 538 individuals who had an acute exacerbation had at least one severe exacerbation, as identified by emergency department visit or hospital admission (appendix). Although exacerbations were more often severe in individuals with greater airflow obstruction, we noted that even in GOLD stage 1 disease, $30(11 \%)$ of 274 individuals had at least one severe event.

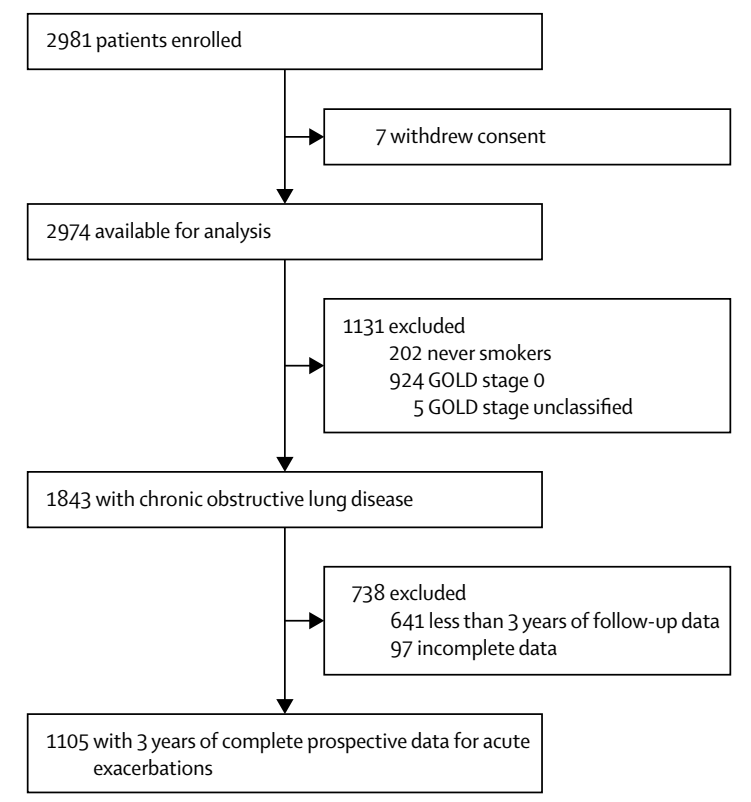

Figure 1: Trial profile

GOLD=Global Initiative for Chronic Obstructive Lung Disease. 


\begin{tabular}{|c|c|c|c|c|}
\hline & \multirow{2}{*}{$\begin{array}{l}\text { All patients } \\
(n=1105)\end{array}$} & \multicolumn{3}{|c|}{ Patients with 3 years of complete acute exacerbation data } \\
\hline & & $\begin{array}{l}\text { Patients with no acute } \\
\text { exacerbation during } \\
\text { follow-up } \\
(n=567,51 \%)\end{array}$ & $\begin{array}{l}\text { Patients with } \\
\text { inconsistent acute } \\
\text { exacerbations } \\
(n=456,41 \%)\end{array}$ & $\begin{array}{l}\text { Patients with at least } \\
\text { one acute exacerbation } \\
\text { in each of the } 3 \text { years } \\
(n=82,7 \%)\end{array}$ \\
\hline Age (years) & $66 \cdot 0(7 \cdot 6)$ & $66.7(7 \cdot 3)$ & $65.5(7 \cdot 8)$ & $64.4(7.9)$ \\
\hline Male sex & $631(57 \%)$ & $357(63 \%)$ & $238(52 \%)$ & $36(44 \%)$ \\
\hline Female sex & $474(43 \%)$ & $210(37 \%)$ & $218(48 \%)$ & $46(56 \%)$ \\
\hline White & $924(84 \%)$ & $477(84 \%)$ & $379(83 \%)$ & $68(83 \%)$ \\
\hline Current smokers & $325(29 \%)$ & $170(30 \%)$ & $137(30 \%)$ & $18(22 \%)$ \\
\hline Postbronchodilator $\mathrm{FEV}_{1}$ (\% predicted) & $63.27(22 \cdot 72)$ & $71.37(20.84)$ & $56 \cdot 33(21.63)$ & $45.91(18.23)$ \\
\hline Acute exacerbation rate in year before enrolment & $0.40(0.87)$ & $0.17(0.54)$ & $0.55(0.96)$ & $1 \cdot 21(1 \cdot 40)$ \\
\hline Acute exacerbation rate in year 1 & $0.37(0.86)$ & $0(0)$ & $0.50(0.84)$ & $2 \cdot 17(1 \cdot 38)$ \\
\hline One or more acute exacerbations in preceding year & $266(24 \%)$ & $66(12 \%)$ & $149(33 \%)$ & $51(62 \%)$ \\
\hline Two or more acute exacerbations in preceding year & $106(10 \%)$ & $15(3 \%)$ & $65(14 \%)$ & $26(32 \%)$ \\
\hline $\begin{array}{l}\text { Severe acute exacerbation requiring admission to } \\
\text { hospital }\end{array}$ & $268(24 \%)$ & $2(<1 \%)$ & $214(47 \%)$ & $54(66 \%)$ \\
\hline COPD assessment test score & $14.29(7.62)$ & $12.05(7.13)$ & $16.06(7.29)$ & $19.68(7 \cdot 40)$ \\
\hline History of gastro-oesophageal reflux disease at baseline & $349(32 \%)$ & $165(29 \%)$ & $155(34 \%)$ & $29(35 \%)$ \\
\hline Chronic bronchitis & $232(21 \%)$ & $96(17 \%)$ & $106(23 \%)$ & $30(37 \%)$ \\
\hline Pi10 & $3 \cdot 71(3 \cdot 66-3 \cdot 78)$ & $3.71(3 \cdot 66-3 \cdot 78)$ & $3 \cdot 71(3 \cdot 65-3 \cdot 78)$ & $3 \cdot 72(3 \cdot 67-3 \cdot 78)$ \\
\hline PRM emphysema & $3(1-13)$ & $2(0-7)$ & $6(1-16)$ & $11(3-25)$ \\
\hline PRM functional small airways disease & $25(15-36)$ & $21(13-31)$ & $31(18-39)$ & $35(29-40)$ \\
\hline Eosinophil count $\left(\times 10^{9}\right.$ per $\left.\mathrm{L}\right)$ & $0.2(0.1-0.3)$ & $0.2(0.1-0.3)$ & $0.2(0.1-0.3)$ & $0.2(0.1-0.3)$ \\
\hline White blood cell count $\left(\times 10^{9}\right.$ per $\left.\mathrm{L}\right)$ & $6 \cdot 9(5 \cdot 8-8 \cdot 20)$ & $6 \cdot 7(5 \cdot 6-8 \cdot 10)$ & $7.05(6.03-8.32)$ & $7 \cdot 35(6 \cdot 3-9 \cdot 20)$ \\
\hline
\end{tabular}

Data are mean (SD), $\mathrm{n}(\%)$, or median (IQR). COPD=chronic obstructive pulmonary disease. Pi10=square root of wall area of a hypothetical airway with an internal perimeter of $10 \mathrm{~mm}$. PRM=parametric response mapping.

Table 1: Baseline characteristics of study participants

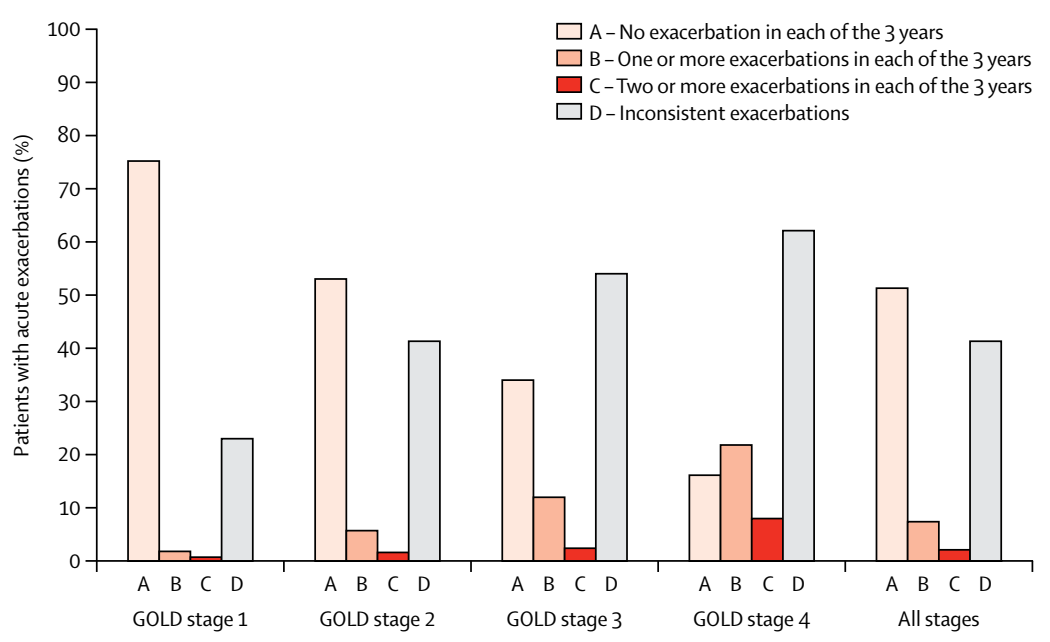

Figure 2: Frequency of acute exacerbations in each of the 3 years in patients with chronic obstructive pulmonary disease

Data are the proportion of patients with each category of acute exacerbation frequency, by GOLD stage and in the entire group ( $\mathrm{n}=1105)$. GOLD=Global Initiative for Chronic Obstructive Lung Disease.

Furthermore, among GOLD stage 1 patients who had at least one exacerbation, $30(44 \%)$ of 68 had at least one exacerbation that was severe (appendix).
The patterns of acute exacerbations over 3 years showed noticeable year to year heterogeneity (figure 2). The most frequent pattern was no exacerbation in any year (567 [51\%] of 1105 patients). The next most common status (456 [41\%] of 1105 patients; appendix) was inconsistent exacerbators who had some years with exacerbations and some years without exacerbations during the 3 years of follow-up. 82 (7\%) patients had at least one exacerbation each year. Only 23 (2\%) of 1105 patients had two or more exacerbations in each of the 3 years of follow-up and would be consistently classified as frequent exacerbators by the ECLIPSE criterion.

Assessment of year-by-year exacerbation status showed that absence of acute exacerbation over 3 years of follow-up was common, and that individuals frequently changed status between years (figure 3). These changes in status do not simply represent acquisition of acute exacerbations in previously exacerbation-free individuals because status changed in both directions. We repeated this analysis restricted to individuals with GOLD 1-2 and 3-4 stage disease and obtained similar results in each instance (appendix). Change in exacerbation pattern from year to year was a common finding in all GOLD groups.

In an analysis restricted to individuals with GOLD 2-4 disease, the pattern of inconsistent acute exacerbations 


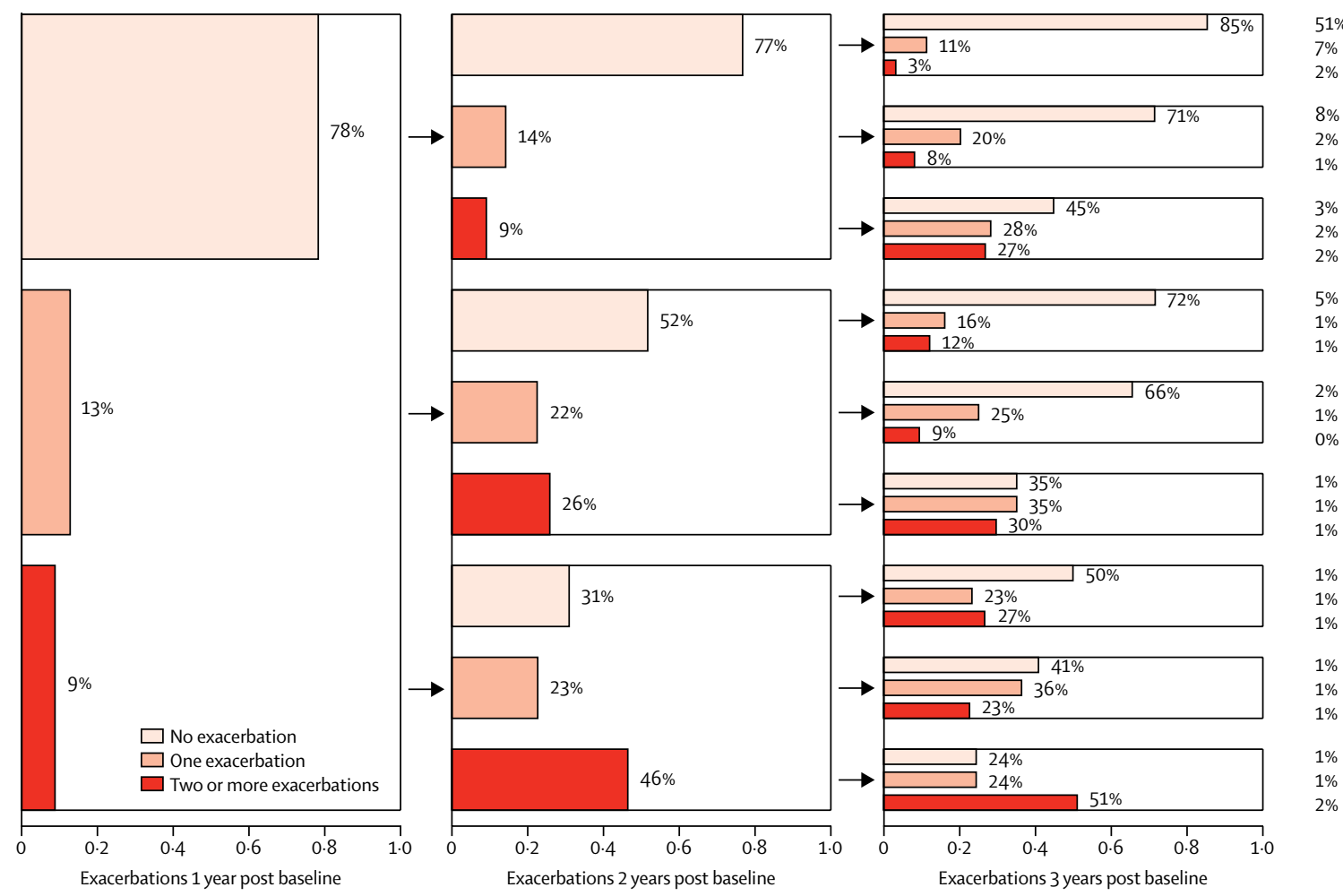

Figure 3: Stability of acute exacerbation frequency patterns over 3 years of prospective follow-up in patients at Global Initiative for Chronic Obstructive Lung Disease stages 1-4

The proportions of participants with no, one, or two or more acute exacerbations in the first year of follow-up are sequentially subdivided by their exacerbation frequency in each of the subsequent years. The final column is the proportion, out of all participants, in the final category.

was most frequent, while 361 (43\%) of 831 participants did not have any exacerbations during 3 years of follow-up (appendix). A distinct minority (21 [3\%] of 831 patients) fell into a consistent frequent exacerbator category throughout the 3 years of follow-up. When the SPIROMICS analysis was limited to GOLD 2-4 participants, between years 1 and 2, 46 (52\%) of 88 frequent exacerbators became infrequent exacerbators, and 103 (14\%) of 743 infrequent exacerbators became frequent exacerbators (appendix). Although exacerbations were more common in individuals with more severe airflow o bstruction, inconsistent a cute exacerbations was the most common status even in GOLD categories 3 and 4 (256 [56\%] of those with inconsistent exacerbations were at GOLD stage 3 or 4), followed by no events. Only $13(4 \%)$ of 337 patients in these categories were characterised as frequent exacerbators (ie, two or more exacerbations per year) during each year of follow-up (appendix).

Due to staggered recruitment, some participants were recruited later and did not have an opportunity to provide 3 full years of follow-up. Given subtle baseline differences between the 1105 individuals with complete 3 years of follow-up data and the 738 for whom data were incomplete, we did a similar analysis combining retrospectively reported acute exacerbations in the year before study entry with the first 2 years of follow-up. With this approach, complete data for 3 years (one retrospective and two prospective) were available for 1471 patients. Again, the pattern of exacerbation frequency was remarkably similar to that of the entirely prospective dataset (appendix). Thus, in this cohort, most individuals showed either a consistent pattern of no acute exacerbation or an inconsistent pattern of variation from year to year, with relatively few having consistent acute exacerbations each year.

The various clinical characteristics of the patient groups with differing patterns of exacerbations during follow-up are in table 1 and the appendix. To identify clinical or biological characteristics associated with consistent acute exacerbations over time, we first compared individuals with at least one exacerbation in every year during 3 years of follow-up (consistent exacerbators) to those who had no exacerbations during follow-up by stepwise logistic regression. Variables associated with patients who consistently had acute exacerbations included increased CAT score, previous acute exacerbations, increased CT-defined small airway abnormality, reduced circulating interleukin-15 concentrations, and increased interleukin-8 concentrations (table 2). $\mathrm{FEV}_{1}$ percentage predicted was significantly associated with consistent exacerbations (table 2). Blood eosinophil count did not predict exacerbation group in any analyses (data not shown). Visual CT analysis was available in 286 individuals. When 


\begin{tabular}{llc} 
& Odds ratio $(95 \% \mathrm{Cl})$ & p value \\
\hline Age & $0.82(0.45-1.50)$ & 0.52 \\
Female sex & $1.41(0.66-3.04)$ & 0.38 \\
Race (white vs other) & $0.70(0.25-2.00)$ & 0.51 \\
Current smoking & $0.62(0.23-1.63)$ & 0.33 \\
FEV $_{1}$ (\% predicted) & $0.80(0.64-1.00)$ & 0.0511 \\
COPD assessment test score & $1.11(1.06-1.17)$ & $<0.0001$ \\
Acute exacerbations in the & $5.22(2.38-11.48)$ & $<0.0001$ \\
year before baseline & & \\
PRM functional small & $1.51(1.07-2.14)$ & 0.0197 \\
airways disease & & \\
Interleukin $15(\mathrm{ng} / \mathrm{mL})$ & $0.04(0.001-0.82)$ & 0.0373 \\
Interleukin $8(\mathrm{pg} / \mathrm{mL})$ & $1.02(1.00-1.04)$ & 0.0460
\end{tabular}

Analysis in 394 patients at Global Initiative for Chronic Obstructive Lung Disease stages 1-4. Percentage predicted $\mathrm{FEV}_{1}$ was re-parameterised by increments of 10 percentage points. Model also adjusted for clinical centre of recruitment. When only the confounders were used (ie, site, age, sex, race, current smoking, $\mathrm{FEV}_{1} \%$ predicted), area under curve $=0.84(95 \% \mathrm{Cl} 0.80-0.89)$. When the full model was used, area under curve $=0.92(95 \% \mathrm{Cl} 0 \cdot 88-0.95)$. COPD=chronic obstructive pulmonary disease. $\mathrm{PRM}$ =parametric response mapping.

Table 2: Results of stepwise logistic regression analysis of one or more exacerbations during each year of 3 years of follow-up versus no exacerbation

tested in the model shown in table 2, visual bronchiectasis was not associated with consistent exacerbations $(p=0 \cdot 58)$.

In a separate analysis, we used a step-wise, zero-inflated negative binomial regression model to assess predictors of exacerbation rate using all patients with any available follow-up data (appendix). As with the logistic model assessing associations with consistent exacerbations, increased CAT score and previous exacerbation history were significantly associated with exacerbation rate (appendix). However, in this analysis, female sex, CTbased air trapping, and increased vascular cell adhesion molecule 1 (VCAM-1) concentrations were also associated with increased exacerbation rate during follow-up. Although $\mathrm{FEV}_{1}$ percentage predicted was associated with exacerbation rate in univariate analysis, it was not significant in the multivariate analysis (appendix). We also ran a subgroup analysis using only frequent exacerbators in the zero-inflated negative binomial model to identify the relationship between eosinophil count and exacerbations. No significant effect for eosinophils was noted $(\mathrm{p}=0.16$ full model adjusted for covariates [appendix] and $\mathrm{p}=0 \cdot 10$ for eosinophils alone). In fact, we noted a nominally decreasing risk of exacerbations (incident rate ratio $<1$ ) as eosinophil count increased.

We also did analyses to assess treatment received for exacerbations. We assessed the first event among those participants who had at least one acute exacerbation in the first year and who had complete data for 3 years of follow-up. Among the individuals in the inconsistent exacerbators group, 38 (32\%) of 120 received antibiotics only, eight (7\%) of 120 systemic steroids only, and 54 (45\%) of 120 received both antibiotics and systemic steroids. Among individuals in the consistent exacerbators group,
13 (29\%) of 45 received antibiotics only, three (7\%) of 45 received steroids only, and 29 (64\%) of 45 received both. To understand how treatment might vary from event to event, we also compared the first treated event in year 1 with the first treated event in year 2. Significant variation in treatment is evident (appendix), but among the 30 individuals who received both antibiotics and steroids for the first event, a large number received both treatments again for the second event $(n=19,63 \%)$.

\section{Discussion}

In a large cohort of highly characterised participants with a broad range of lung function impairment, we report that the most durable acute exacerbation phenotype is the absence of events over a 3-year period, reported in $51 \%$ of individuals. Among participants who had at least one exacerbation over 3 years, exacerbation status was highly variable, with only $7 \%$ of the cohort consistently having at least one exacerbation each year, and only $2 \%$ having two or more exacerbations in each year. When the analysis was limited to GOLD 3 and GOLD 4 status, only $1 \%$ of participants had two or more exacerbations in every year. In multivariate analysis, consistent exacerbations, defined as one or more acute exacerbations per year in every year of follow-up, were associated with higher CAT score, previous history of exacerbations, CT-defined small airway abnormality, lower circulating interleukin- 15 concentrations, and higher circulating interleukin-8 concentrations.

In this study, only $2 \%$ of COPD participants had two or more exacerbations in each of 3 years of follow-up. Even in the more severe ECLIPSE cohort, only $12 \%$ of patients consistently had two or more exacerbations per year during 3 years of follow-up. ${ }^{17}$ Data from the ECLIPSE study suggest that patients with a history of two or more exacerbations in a previous year represent a relatively stable frequent-exacerbator phenotype associated with persistently increased inflammation. ${ }^{4}$ However, between years 1 and 2, 210 (39\%) of 543 patients changed from frequent exacerbators $(\geq 2$ acute exacerbations) to infrequent exacerbators (0-1 acute exacerbation), while 221 (17\%) of 1289 changed from infrequent exacerbators to frequent exacerbators. ${ }^{17}$ When the SPIROMICS analysis was limited to GOLD 2-4 participants, between years 1 and 2, 46 (52\%) of 88 frequent exacerbators became infrequent exacerbators, while 103 (14\%) of 473 infrequent exacerbators became frequent exacerbators (appendix). In a smaller study, Brusse-Keizer and colleagues ${ }^{18}$ also reported on exacerbation frequency in a moderate to severe COPD cohort of 121 patients. Similar to SPIROMICS, between enrolment and year 1, 21 (62\%) of 34 frequent exacerbators changed to infrequent exacerbators, while $18(21 \%)$ of 87 infrequent exacerbators changed to frequent exacerbators. Although these various populations were recruited by separate investigative groups during different time periods, necessitating caution in making direct comparisons, they show the regularity with which individuals change exacerbation categories. 
In the SPIROMICS cohort, we also show an association between consistent acute exacerbations and increased functional small airway abnormality and parametric response mapping functional small airways disease, as detected via recently developed CT metrics. This abnormality has also previously been identified a $\mathrm{s}$ a marker of more rapid lung function decline in COPD. ${ }^{3}$ Previous analyses of exacerbations have shown an association between segmental level wall thickness measured at the fourth generation and exacerbations, but parametric response mapping functional small airways disease was not included in that analysis. ${ }^{19}$ In the present study, we noted that parametric response mapping functional small airways disease was strongly associated with consistent exacerbations. Associations between low interleukin-15 concentrations and high interleukin- 8 concentrations and consistent exacerbations were also reported. In a a zero-inflated negative binomial model, previous acute exacerbations, CAT score, and percentage gas trapping on CT (another indirect measure of small airway abnormality) were associated with exacerbation count, similar to the first model. However, several other significant associations emerged, including female sex and high levels of circulating VCAM-1. Concentrations of interleukin 15 and interleukin 8 were not significant in this alternative model nor was $\mathrm{FEV}_{1}$ percentage predicted. Hence, it is plausible that the characteristics of patients with consistent exacerbations are different from those of people with inconsistent exacerbations. Interestingly, although $\mathrm{FEV}_{1}$ percentage predicted was important in the univariate analysis and exacerbations were more common in patients with more severe airflow obstruction, multivariate analyses yielded limited evidence to support an independent contribution of this parameter. In two different multivariate analyses, $\mathrm{FEV}_{1}$ percentage predicted met the 0.05 significance level in one but was not reported to be significant in the other. These finding a re probably a function of close interaction between that parameter and other important patient characteristics, such as CT features of COPD.

Ultimately these data have implications for stratification of patients both in clinical practice and for research. Frequent exacerbator status defined a $\mathrm{st}$ wo o $\mathrm{r} \mathrm{m}$ ore exacerbations in every year is distinctly uncommon; in our cohort, only $2 \%$ of patients at GOLD 1-4 disease status and $1 \%$ of patients at GOLD 3-4 status were in this category. This variability in yearly exacerbation rates could stem from failure to consider the multiple triggers that initiate exacerbations. Whether an individual patient encounters a potent trigger for exacerbation within any given year might determine whether or not that individual has an exacerbation in that year. Present GOLD stratification schema use a history of two or more exacerbations in the previous year as one way to identify those at increased risk of future events. ${ }^{5}$ The frequency of these events and their consistency across a broad range of patient groups has not been thoroughly assessed. Although our data support a relationship between previous and future exacerbations, they also indicate that exacerbation frequency is highly variable over time. Among individuals who inconsistently exacerbate, factors extrinsic to the individual, such as specific environmental or occupational exposures, might have a strong role in exacerbation occurrence, making these events difficult to predict.

We acknowledge limitations to this analysis. This cohort is not population-based and therefore might be biased, as the types of patients evaluated at academic centres might differ from the general COPD patient population. By design, this cohort also has more mildly affected individuals than other cohort studies, such as ECLIPSE in which only GOLD 2-4 individuals were included. Decisions about treatment of COPD were made by the patients' own physicians and were not guided by study protocol. Such analyses also might differ based on the types of exacerbations studied. Here, we chose to assess moderate to severe events requiring a health-care visit. Daily diary data from the Exact Pro instrument was captured in a subset of individuals and will be assessed in future analyses. Strengths of this study, however, include rigorous data collection through systematic and frequent contacts with participating patients; inclusion of participants exhibiting a wide range of disease severity; and detailed phenotyping including $\mathrm{CT}$ and blood biomarkers.

We report that in a COPD cohort (GOLD 1-4) not selected for recent exacerbations, acute exacerbation frequency varied greatly from year to year. The two most common phenotypes were no exacerbations over 3 years $(51 \%$ of patients) and the inconsistent exacerbator, who changed exacerbation status from year to year (41\% of patients). Those with two or more exacerbations in every year represented only approximately $2 \%$ of our cohort. We identified a group of individuals (7\% of patients) who consistently exacerbated over time as defined by one or more exacerbations every year during 3 years of follow-up. Among these individuals, in addition to previous exacerbation history and CAT score, we also identified CTdefined small airway abnormality, low interleukin-15 concentrations, and increased interleukin-8 concentrations as predictors of consistent exacerbation status. Among individuals who inconsistently exacerbate, it is plausible that factors beyond the individual, such as exposure to external triggers, have a strong association with exacerbation occurrence, making these events more difficult to predict.

\section{Contributors}

MKH, PMQ, EEC, DJC, RP III, JLC, and FJM contributed to the conceptualisation of the study. MKH, RGB, ERB, RPB, CBC, AC, GC, MTD, NNH, REK, JAK, PW, RP III, and FJM were involved in data collection. MKH, PMQ, EEC, DJC, and FJM contributed to data analysis. All authors participated in manuscript writing and editing.

\section{Declaration of interests}

MKH and RGB received grants from the National Institutes of Health (NIH), and Foundation for the NIH, COPD Foundation, during the study. MKH received consulting fees from Boehringer Ingelheim, GlaxoSmithKline, Novartis, AstraZeneca, and Sunovion, outside of this Article; and royalties 
from UpToDate and research support from Novartis. RGB received grants from Alpha1 Foundation and personal fees from UpToDate, outside of this Article. CBC received grants from Equinox Health Clubs, Amgen, and Spiration; personal fees from Equinox Health Clubs, PulmonX, Boehringer Ingelheim, GlaxoSmithKline, and Spiration, outside of this Article; and works part-time on scientific engagement for the GlaxoSmithKline Global Respiratory Franchise. JLC received grants from NIH and the National Heart, Lung, and Blood Institute (NHLBI), during the study; grants from the National Institute of Allergy and Infectious Diseases, Department of Veterans Affairs, Department of Defense, MedImmune, and COPD Foundation, outside of this Article. MTD received grants from NHLBI, during the study; grants from the Department of Defense, personal fees and other from Boehringer Ingelheim, GlaxoSmithKline, and Boston Scientific; personal fees and other from AstraZeneca, other from Novartis, Yungjin, PneumRx and BTG, and Pulmonx; personal fees from Genentech outside of this Article. EAH is a founder and shareholder of VIDA Diagnostics. SR is employed by AstraZeneca and also retains professorship and a part-time appointment at the University of Nebraska Medical Center (Omaha, NE, USA); received personal fees from ABIM, Able Associates, Advantage Healthcare, Align2Action, Almirall, APT, ATS, AstraZeneca, Baxter, Boehringer Ingelheim, Cheisi, CIPLA, ClearView Healthcare, Cleveland Clinic, CME Incite, Complete Medical Group, COPD Foundation, Cory Paeth, CSA, CSL, CTS Carmel, Dailchi Sankyo, Decision Resources, Dunn Group, Easton Associates, Elevation Pharma, FirstWord, Forest, Frankel Group, Gerson, GlaxoSmithKline, Gilead, Grifols, GroupH, Guidepoint Global, Haymarket, HealthStar, Huron Consulting, Incite, Inthought, IntraMed (Forest), Johnson and Johnson, LEK, McKinsey, Medical Knowledge, MedImmune, Methodist Health System Dallas, Navigant, NCI Consulting, Novartis, Nuvis, Pearl, Penn Technology, Pfizer, PlanningShop, Prescott, Pro Ed Comm, ProiMed, PSL FirstWord, Pulmatrix, Quadrant, Qwessential, Regeneron, Saatchi and Saatchi, Schlesinger Associates, Strategic North, Synapse, Takeda, Theron, and WebMD; and grants from NHLBI, Nebraska Department of Health and Human Services, Otsuka, Pfizer, GlaxoSmithKline, Boehringer Ingelheim, Nycomed, AstraZeneca, grants from Centocor, grants from Almirall, outside of this Article. DPT received personal fees from Boehringer Ingelheim, AstraZeneca, Sunovion, Novartis, Theravance, and Innoviva, outside of this Article. JAW received personal fees and non-financial support from Novartis; grants, personal fees, and non-financial support from GlaxoSmithKline; grants, personal fees, and non-financial support from Takeda; personal fees and non-financial support from AstraZeneca, and Boehringer Ingelheim, outside of the submitted work; and she is the editor of American Journal of Respiratory and Critical Care Medicine. PW received grants from MedImmune, personal fees from Genentech and Roche, AstraZeneca, Novartis, Neostem, and Janssen, outside of this Article; and has a patent Asthma diagnostics pending. FJM received grants from NHLBI, during the study; grants from $\mathrm{NIH}$ and personal fees from Continuing Education, Forest Laboratories, GlaxoSmithKline, Nycomed and Takeda, AstraZeneca, Boehringer Ingelheim, Bellerophon (formerly Ikaria), Genentech, Novartis, Pearl, Roche, Sunovion, Theravance, CME Incite, Annenberg Center for Health Sciences at Eisenhower, Integritas, InThought, National Association for Continuing Education, Paradigm Medical Communications LLC, PeerVoice, UpToDate, Haymarket Communications, Western Society of Allergy and Immunology, Proterixbio (formerly Bioscale), Unity Biotechnology, ConCert Pharmaceuticals, Lucid, New York-Presbyterian Brooklyn Methodist Hospital, Columbia University, Prime Healthcare Ltd, WebMD, PeerView Network, California Society of Allergy and Immunology, Chiesi, and Puerto Rico Thoracic Society, outside of this Article. AC has consulted for VIDA Diagnostics. DJC reports grants from NHLBI of the NIH, during the study; and grants from COPD Foundation, outside of the Article. NNH received grants and personal fees from AstraZeneca and GlaxoSmithKline, grants from Boehringer Ingelheim, NIH, and COPD Foundation, outside of the Article. JAK received grants from NIH and Patient Centered Outcomes Research Institute, outside of the Article. RP III received grants from NHLBI, and COPD Foundation, during the study; and grants from Department of Veterans Affairs, outside of the Article. EEC received funding from NHLBI, the Foundation for the NIH, Genentech, and the COPD Foundation, during the study. All other authors declare no competing interests.

\section{Acknowledgments}

For more on SPIROMICS see The authors thank the SPIROMICS participants and participating www.spiromics.org physicians, investigators, and staff for making this research possible. SPIROMICS was supported by contracts from the NIH and NHLBI
(HHSN268200900013C, HHSN268200900014C, HHSN268200900015C HHSN268200900016C, HHSN268200900017C, HHSN268200900018C, HHSN268200900019C, HHSN268200900020C), which were supplemented by contributions made through the Foundation for the NIH from AstraZeneca, Bellerophon Therapeutics, Boehringer Ingelheim Pharmaceuticals Inc, Chiesi Farmaceutici SpA, Forest Research Institute Inc, GlaxoSmithKline, Grifols Therapeutics Inc, Ikaria Inc, Nycomed $\mathrm{GmbH}$, Takeda Pharmaceutical Company, Novartis Pharmaceuticals Corporation, Regeneron Pharmaceuticals Inc, and Sanofi. This analysis was also supported by NHLBI R01 HL122438 and HL126838.

\section{References}

1 Jones PW. St. George's respiratory questionnaire: MCID. COPD 2005; 2: 75-79.

2 Seemungal TA, Donaldson GC, Paul EA, Bestall JC, Jeffries DJ, Wedzicha JA. Effect of exacerbation on quality of life in patients with chronic obstructive pulmonary disease. Am J Respir Crit Care Med 1998; 157: 1418-22.

3 Donaldson GC, Seemungal TA, Bhowmik A, Wedzicha JA. Relationship between exacerbation frequency and lung function decline in chronic obstructive pulmonary disease. Thorax 2002; 57: 847-52.

4 Bhatt SP, Soler X, Wang X, et al. Association between functional small airways disease and FEV1 decline in COPD. Am J Respir Crit Care Med 2016; 194: 178-84.

5 McGhan R, Radcliff T, Fish R, Sutherland ER, Welsh C, Make B. Predictors of rehospitalization and death after a severe exacerbation of COPD. Chest 2007; 132: 1748-55.

6 Buist AS, Vollmer WM, McBurnie MA. Worldwide burden of COPD in high- and low-income countries. Part I. The burden of obstructive lung disease (BOLD) initiative. Int J Tuberc Lung Dis 2008; 12: 703-08.

7 Pavord ID, Jones PW, Burgel PR, Rabe KF. Exacerbations of COPD. Int J Chron Obstruct Pulmon Dis 2016; 11: 21-30.

8 Hurst JR, Vestbo J, Anzueto A, et al. Susceptibility to exacerbation in chronic obstructive pulmonary disease. N Engl J Med 2010; 363: 1128-38.

9 Vestbo J, Hurd SS, Agustí AG, et al. Global strategy for the diagnosis management, and prevention of chronic obstructive pulmonary disease: GOLD executive summary. Am J Respir Crit Care Med 2013; 187: 347-65.

10 Couper D, LaVange LM, Han M, et al. Design of the Subpopulation and Intermediate Outcomes in COPD Study (SPIROMICS). Thorax 2014; 69: 491-94.

11 Hankinson JL, Odencrantz JR, Fedan KB. Spirometric reference values from a sample of the general U.S. population. Am J Respir Crit Care Med 1999; 159: 179-87.

12 Sturrock A, Seedahmed E, Mir-Kasimov M, Boltax J, McManus ML Paine R 3rd. GM-CSF provides autocrine protection for murine alveolar epithelial cells from oxidant-induced mitochondrial injury. Am J Physiol Lung Cell Mol Physiol 2012; 302: L343-51.

13 Sieren JP, Newell JD Jr, Barr RG, et al. SPIROMICS protocol for multicenter quantitative computed tomography to phenotype the lungs. Am J Respir Crit Care Med 2016; 194: 794-806.

14 Patel BD, Coxson HO, Pillai SG, et al. Airway wall thickening and emphysema show independent familial aggregation in chronic obstructive pulmonary disease. Am J Respir Crit Care Med 2008; 178: $500-05$.

15 Galban CJ, Han MK, Boes JL, et al. Computed tomography-based biomarker provides unique signature for diagnosis of COPD phenotypes and disease progression. Nat Med 2012; 18: 1711-15.

16 Jones PW, Harding G, Berry P, Wiklund I, Chen WH, Kline Leidy N. Development and first validation of the COPD assessment test. Eur Respir J 2009; 34: 648-54.

17 Donaldson GC, Mullerova H, Locantore N, et al. Factors associated with change in exacerbation frequency in COPD. Respir Res 2013; 14: 79.

18 Brusse-Keizer M, van der Palen J, van der Valk P, Hendrix R, Kerstjens H. Clinical predictors of exacerbation frequency in chronic obstructive pulmonary disease. Clin Respir J 2011; 5: 227-34.

19 Han MK, Kazerooni EA, Lynch DA, et al. Chronic obstructive pulmonary disease exacerbations in the COPDGene study: associated radiologic phenotypes. Radiology 2011; 261: 274-82. 